\title{
Bifurcation Dynamics in a Fractional-Order Oregonator Model Including Time Delay
}

\author{
Changjin $\mathrm{Xu}^{1}$, Wei Zhang ${ }^{2}$, Chaouki Aouiti ${ }^{3}$, Zixin $\mathrm{Liu}^{4}$, \\ Peiluan $\mathrm{Li}^{5}$, Lingyun $\mathrm{Yao}^{6}$ \\ ${ }^{1}$ Guizhou Key Laboratory of Economics System Simulation \\ Guizhou University of Finance and Economics, Guiyang 550004, PR China \\ $\mathrm{xcj}$ 403@126. com \\ 2 College of Economics and Statistics, Guangzhou University \\ Guangzhou 510006, PR China \\ wzha19680317@126.com \\ ${ }^{4}$ Faculty of Sciences of Bizerta, UR13ES47 Research Units of Mathematics \\ and Applications, University of Carthage, Bizerta 7021, Tunisia \\ chaouki.aouiti@fsb.rnu.tn \\ ${ }^{4}$ School of Mathematics and Statistics
}

Guizhou University of Finance and Economics, Guiyang 550004, PR China xinxin905@126.com

${ }^{5}$ School of Mathematics and Statistics, Henan University of Science and Technology Luoyang 471023, PR China lpllpl_lpl@163.com

${ }^{6}$ Library, Guizhou University of Finance and Economics, Guiyang 550004, PR China lingyunyao2015@126.com

(Received July 31, 2021)

\begin{abstract}
Setting up mathematical models to describe the interaction of chemical variables has been a hot issue in chemical and mathematical areas. Nevertheless, many mathematical models are only involved with the integer-order differential equation case. The fruits on fractional-order chemical models are very scarce. In this present work, on the basis of the previous studies, we set up a novel fractional-order delayed Oregonator model. Selecting the time delay as bifurcation parameter, we obtain novel delay-independent bifurcation conditions that guarantee the stability
\end{abstract}


and the appearance of Hopf bifurcation for the fractional-order delayed Oregonator model. The study shows that time delay plays a vital role in controlling the stability and the appearance of Hopf bifurcation of the considered fractional-order delayed Oregonator model. In order to verify the rationality of theoretical results, computer simulations are carried out.

\section{Introduction}

In various chemical systems, feedback has an important effect on their dynamics. In particular, time delayed feedback on the chemical systems has received much interest from many researchers. Since the work of Belousov [1], the Belousov Zhabotinsky (BZ) system has attracted great attention from many scholars (see [2,3]). Oregonator model is an important model that describes the BZ chemical reaction. Usually, Oregonator model is a nonlinear dynamical system. In 1994, Gray and Scott [4] proposed the following Oregonator model:

$$
\left\{\begin{array}{l}
\frac{d \mathcal{X}(t)}{d t}=\kappa_{3} \mathcal{A} \mathcal{Y}-\kappa_{2} \mathcal{X Y}+\kappa_{5} \mathcal{A} \mathcal{X}-2 \kappa_{4} \mathcal{X}^{2} \\
\frac{d \mathcal{Y}(t)}{d t}=\kappa_{3} \mathcal{A} \mathcal{Y}-\kappa_{2} \mathcal{X} \mathcal{Y}+\frac{1}{2} g \kappa_{0} \mathcal{B Z} \\
\frac{d \mathcal{Z}(t)}{d t}=2 \kappa_{5} \mathcal{A} \mathcal{X}-\kappa_{0} \mathcal{B Z}
\end{array}\right.
$$

where $\mathcal{X}=\left[\mathrm{HBrO}_{2}\right]$ denotes the concentrations of $\mathrm{HBrO}_{2}, \mathcal{Y}=\left[\mathrm{Br}^{-}\right]$denotes the concentrations of $\mathrm{Br}^{-}, \mathcal{Z}=[\mathrm{Ce}(\mathrm{IV})]$ denotes the concentrations of $\mathrm{Ce}(\mathrm{IV}), \mathcal{A}=\left[\mathrm{BrO}_{3}^{-}\right]=$ $\mathcal{B}$ denotes the concentrations of $\mathrm{BrO}_{3}^{-}, \kappa_{0}, \kappa_{3}, \kappa_{4}, \kappa_{5}$ are positive constants and $g$ stands for a changeable parameter. The dimensional version of system (1) takes the form:

$$
\left\{\begin{array}{l}
\epsilon \frac{d w_{1}(t)}{d t}=a w_{2}-w_{1} w_{2}+w_{1}\left(1-w_{1}\right) \\
\rho \frac{d w_{2}(t)}{d t}=-a w_{2}-w_{1} w_{2}+g w_{3} \\
\frac{d w_{3}(t)}{d t}=w_{1}-w_{3},
\end{array}\right.
$$

where $\epsilon, \rho, a, g$ are positive constants. In [5], Wu and Zhang pointed out that $\rho \ll \epsilon$, then $\rho \frac{d w_{2}(t)}{d t} \rightarrow 0$ which leads to $w_{2} \approx \frac{g w_{3}}{a+w_{1}}$. Substituting $w_{2} \approx \frac{g w_{3}}{a+w_{1}}$ into the first equation and the third equation of system (2), we get

$$
\left\{\begin{array}{l}
\epsilon \frac{d w_{1}(t)}{d t}=\frac{a g w_{3}}{a+w_{1}}-\frac{g w_{1} w_{3}}{a+w_{1}}+w_{1}\left(1-w_{1}\right), \\
\frac{d w_{3}(t)}{d t}=w_{1}-w_{3},
\end{array}\right.
$$

Considering the effect of electric current, the concentrations of $C e(I V)$ will be affected by the past time. Thus there exists a time delay of self-feedback. In order to describe the 
perturbation, Wu and Zhang [5] introduced the perturbation term $\theta w_{3}(t-\gamma)$ into system (3) and obtained the following system:

$$
\left\{\begin{array}{l}
\epsilon \frac{d w_{1}(t)}{d t}=\frac{a g w_{3}}{a+w_{1}}-\frac{g w_{1} w_{3}}{a+w_{1}}+w_{1}\left(1-w_{1}\right), \\
\frac{d w_{3}(t)}{d t}=w_{1}-w_{3}+\theta w_{3}(t-\gamma),
\end{array}\right.
$$

where $\gamma$ is a time delay and $\theta$ is a control parameter. By applying the stability theorem and bifurcation theory of delayed differential equation, Wu and Zhang [5] set up a delayindependent condition to ensure the stability and the existence of Hopf bifurcation for integer-order delayed system (3).

In recent years, fractional calculus, which is regarded as a generalization of ordinary differentiation and integration, has attracted much attention from many scholars due to its great application prospect in numerous fields such as chemistry, neural networks, viscoelasticity, biological system, physics, economics and so on [6-8]. The study shows that fractional-order differential equation is a more suitable tool to describe the actual phenomenon in natural world than the integer-order ones since fractional-order differential equation depicts the memory and hereditary trait of all kinds of materials and physical process [9]. At present, a lot of researchers concentrate on the dynamical nature of fractional-order dynamical models and rich fruits has already borne. One can refer to [10-13]. Hopf bifurcation is an important dynamical phenomenon of fractional-order delayed dynamical systems. Revealing the impact of time delay on Hopf bifurcation of delayed dynamical models is a crucial topic in fractional-order delayed differential equation. Nowadays some valuable fruits on fractional-order differential systems have been achieved. For example, Xu et al. [14] reported the Hopf bifurcation of fractional-order BAM neural networks including multiple time delays; Yuan et al. [15] studied the stability and Hopf bifurcation for a fractional-order prey-predator system including two different time delays; Eshaghi et al. [16] investigated the Hopf bifurcation, chaos control and synchronization issue for a chaotic fractional-order model. In details, one can see [17-25].

However, it is a pity that all the above works on Hopf bifurcation of fractional-order dynamical systems merely focus on predator-prey models and neural networks. Up to now, there are very few publications that are concerned with chemical systems. In order to make up for deficiency, we think that it is necessary to probe into the delay-induced Hopf bifurcation issue for the fractional-order Oregonator model. Inspired by the viewpoint 
and based on system (4), we revise system (4) as the following delayed Oregonator model:

$$
\left\{\begin{array}{l}
\epsilon \frac{d w_{1}^{\eta}(t)}{d t^{\eta}}=\frac{a g w_{3}}{a+w_{1}}-\frac{g w_{1} w_{3}}{a+w_{1}}+w_{1}\left(1-w_{1}\right), \\
\frac{d w_{3}^{\eta}(t)}{d t^{\eta}}=w_{1}-w_{3}+\theta w_{3}(t-\gamma),
\end{array}\right.
$$

where $\eta \in(0,1]$ and all the parameters owns the same implication as those in system (4). In details, one can see [5]. We assume that the initial values of system (5) is $w_{j}(t)=\varphi_{j}(t)(j=1,2)$, where $\varphi_{j}(t) \in[-\gamma, 0]$. We think that fractional order model (5) has greater advantages than the integer-order ones since it can better describe the continuous transformation process of the the concentrations of $\mathrm{HBrO}_{2}$ and the concentrations of $\mathrm{BrO}_{3}^{-}$in chemistry.

The key aim of this work can be stated as follows: (1) setting up the delay-independent conditions that guarantee the stability and emergence of Hopf bifurcation for Oregonator model (5); (2) The influence of time delay on Hopf bifurcation of Oregonator model (5) is sufficiently revealed.

The key contribution of this work can be summarized as follows:

- Based on the previous research, we set up a novel fractional-order regonator model that can describe the memory and hereditary trait of the concentrations of $\mathrm{HBrO}_{2}, \mathrm{Br}^{-}$and $C e(I V)$.

- A novel delay-independent criterion guaranteeing the stability and the emergence of Hopf bifurcation of fractional-order delayed Oregonator model is derived. The influence of time delay on the stability and Hopf bifurcation of fractional-order delayed Oregonator model (1.5) is explained.

- Up to now, few authors deal with the Hopf bifurcation of fractional-order delayed Oregonator model .

The rest of this work is arranged as follows. Part 2 presents some necessary knowledge on fractional-order dynamical system. Part 3 gives delay-independent criterion guaranteeing the stability and the emergence of Hopf bifurcation of fractional-order delayed Oregonator model. Also, the influence of time delay on the stability and Hopf bifurcation of fractional-order delayed Oregonator model (5) is presented. Part 4 carries out matlab simulation to check the key theoretical fruits. Part 4 finishes this work. 


\section{Preliminary knowledge}

In this part, several essential definitions and lemmas on fractional-order differential equation are prepared.

Definition 2.1. [26] Define the fractional integral of order $\eta$ of the function $l(\zeta)$ as follows:

$$
\mathcal{I}^{\eta} l(\zeta)=\frac{1}{\Gamma(\eta)} \int_{\zeta_{0}}^{\zeta}(\zeta-s)^{\eta-1} l(s) d s,
$$

where $\zeta \geq \zeta_{0}, \eta>0$, and $\Gamma(s)=\int_{0}^{\infty} \zeta^{s-1} e^{-\zeta} d \zeta$ represents Gamma function.

Definition 2.2. [26] Assume that $l(\zeta) \in C\left(\left[\zeta_{0}, \infty\right), R\right)$. The Caputo-type fractionalorder derivative of order $\eta$ of $l(\zeta)$ is given by

$$
\mathcal{D}^{\eta} l(\zeta)=\frac{1}{\Gamma(l-q)} \int_{\zeta_{0}}^{\zeta} \frac{l^{(h)}(s)}{(\xi-s)^{\eta-h+1}} d s,
$$

where $\zeta \geq \zeta_{0}$ and $h$ stands for a positive integer satisfying $h-1 \leq \eta<1$. In addition, if $0<\eta<1$, then

$$
\mathcal{D}^{\eta} l(\zeta)=\frac{1}{\Gamma(1-\eta)} \int_{\zeta_{0}}^{\zeta} \frac{l^{\prime}(s)}{(\zeta-s)^{\eta}} d s .
$$

Definition 2.3. [27] For the following fractional-order system:

$$
\mathcal{D}^{\eta} w_{i}(t)=g_{i}\left(w_{i}(t)\right), i=1,2, \cdots, k,
$$

where $\eta \in(0,1], w_{i}(t)=\left(w_{1}(t), w_{2}(t), \cdots, w_{k}(t)\right), g_{i}(t)=\left(g_{1}(t), g_{2}(t), \cdots, g_{k}(t)\right)$. We say that $\left(w_{1}^{*}, w_{2}^{*}, \cdots, w_{k}^{*}\right)$ is the equilibrium point provided that $g_{i}\left(w_{i}^{*}\right)=0$.

Lemma 2.1. [28] Consider the fractional-order system $\mathcal{D}^{\eta} u=\mathcal{B} u, u(0)=u_{0}$ where $0<\eta<1, u \in R^{m}, \mathcal{B} \in R^{m \times m}$. Suppose that $\lambda_{i}(i=1,2, \cdots, m)$ is the root of the characteristic equation of $\mathcal{D}^{\eta} u=\mathcal{B} u$. Then system $\mathcal{D}^{\eta} u=\mathcal{B} u$ is said to be asymptotically stable $\Leftrightarrow\left|\arg \left(\lambda_{j}\right)\right|>\frac{\eta \pi}{2}(j=1,2, \cdots, m)$. In addition, this system is said to be stable $\Leftrightarrow$ $\left|\arg \left(\lambda_{j}\right)\right|>\frac{\eta \pi}{2}(j=1,2, \cdots, m)$ and those critical eigenvalues satisfying $\left|\arg \left(\lambda_{i}\right)\right|=\frac{\eta \pi}{2}(j=$ $1,2, \cdots, m)$ own geometric multiplicity one.

Lemma 2.2. [29] Consider the fractional-order system $\mathcal{D}^{\eta} w(t)=\mathcal{P}_{1} w(t)+\mathcal{P}_{2} w(t-\gamma)$, where $w(t)=\chi(t), t \in[-\gamma, 0], \eta \in(0,1], w \in R^{m}, \mathcal{P}_{1}, \mathcal{P}_{2} \in R^{m \times m}, \varrho \in R^{+(m \times m)}$. Then the characteristic equation of the system takes the form: $\operatorname{det}\left|s^{q} \mathcal{I}-\mathcal{P}_{1}-\mathcal{P}_{2} e^{-s \gamma}\right|=0$. Then the zero solution of the system is said to be asymptotically stable provided that every root of the characteristic equation of the system owns negative real roots. 


\section{Bifurcation exploration for system (5)}

In this section, we will discuss the impact of time delay on Hopf bifurcation for model (5).

Denote $\left(w_{1 *}, w_{3 *}\right)$ the equilibrium point of system (3), then

$$
\left\{\begin{array}{l}
\frac{a g w_{3 *}}{a+w_{1 *}}-\frac{g w_{1 *} w_{3 *}}{a+w_{1 *}}+w_{1 *}\left(1-w_{1 *}\right)=0 \\
w_{1 *}-w_{3 *}+\theta w_{3 *}=0
\end{array}\right.
$$

which leads to

$$
\left\{\begin{array}{l}
w_{1 *}=\frac{1-\frac{g}{1-\theta}-a+\sqrt{\left(1-\frac{g}{1-\theta}-a\right)^{2}+4 a\left(1+\frac{g}{1-\theta}\right)}}{2}, \\
w_{3 *}=\frac{w_{1 *}}{1-\theta} .
\end{array}\right.
$$

Clearly, system (5) owns the unique positive equilibrium point $\left(w_{1 *}, w_{3 *}\right)$ if the following condition

$$
\left(A_{1}\right) \quad \theta<1
$$

holds. Denote $\bar{w}_{1}(t)=w_{1}(t)-w_{1 *}, \bar{w}_{3}(t)=w_{3}(t)-w_{3 *}$, then system (5) can be expressed as the form:

$$
\left\{\begin{aligned}
\frac{d \bar{w}_{1}^{\eta}(t)}{d t^{\eta}}= & \frac{1}{\epsilon}\left[\frac{a g\left(\bar{w}_{3}+w_{3 *}\right)}{a+\bar{w}_{1}+w_{1 *}}-\frac{g\left(\bar{w}_{1}+w_{1 *}\right)\left(\bar{w}_{3}+w_{3 *}\right)}{a+\left(\bar{w}_{1}+w_{1 *}\right)}\right. \\
& \left.+\left(\bar{w}_{1}+w_{1 *}\right)\left(1-\left(\bar{w}_{1}+w_{1 *}\right)\right)\right] \\
\frac{d \bar{w}_{3}^{\eta}(t)}{d t^{\eta}}= & \left(\bar{w}_{1}+w_{1 *}\right)-\left(\bar{w}_{3}+w_{3 *}\right)+\left(\theta \bar{w}_{3}(t-\gamma)+w_{3 *}\right)
\end{aligned}\right.
$$

The linearization of Eq. (9) around $(0,0)$ can be written

$$
\left\{\begin{array}{l}
\frac{d \bar{w}_{1}^{\eta}(t)}{d \eta^{\eta}}=\alpha_{1} \bar{w}_{1}+\alpha_{2} \bar{w}_{3} \\
\frac{d \bar{w}_{3}^{\eta}(t)}{d t^{\eta}}=\bar{w}_{1}-\bar{w}_{3}+\theta \bar{w}_{3}(t-\gamma)
\end{array}\right.
$$

where

$$
\left\{\begin{aligned}
\alpha_{1} & =\frac{1}{\epsilon}\left[+1-2 w_{1 *}-\frac{2 a g w_{3 *}}{\left(a+w_{1 *}\right)^{2}}\right] \\
\alpha_{2} & =\frac{g\left(a-w_{1 *}\right)}{\epsilon\left(a+w_{1 *}\right)}
\end{aligned}\right.
$$

In system (10), we still denote $\bar{w}_{1}, \bar{w}_{3}$ by $w_{1}, w_{3}$, respectively, then system (10) can be rewritten as

$$
\left\{\begin{array}{l}
\frac{d w_{1}^{\eta}(t)}{d t^{\eta}}=\alpha_{1} w_{1}+\alpha_{2} w_{3} \\
\frac{d w_{3}^{\eta}(t)}{d t^{\eta}}=w_{1}-w_{3}+\theta w_{3}(t-\gamma)
\end{array}\right.
$$


The characteristic equation of system (12) is written as:

$$
\operatorname{det}\left[\begin{array}{cc}
s^{\eta}-\alpha_{1} & -\alpha_{2} \\
-1 & s^{\eta}+1-\theta e^{-s \gamma}
\end{array}\right]=0,
$$

which leads to

$$
s^{2 \eta}+\beta_{1} s^{\eta}+\beta_{2}+\left(\varsigma_{1} s^{\eta}+\varsigma_{2}\right) e^{-s \gamma}=0
$$

where

$$
\left\{\begin{array}{l}
\beta_{1}=1-\alpha_{1}, \\
\beta_{2}=-\left(\alpha_{1}+\alpha_{2}\right), \\
\varsigma_{1}=-\theta \\
\varsigma_{2}=\theta \alpha_{1} .
\end{array}\right.
$$

Now the assumption is given as follows:

$$
\left(A_{2}\right) \beta_{1}+\varsigma_{1}>0, \beta_{2}+\varsigma_{2}>0 .
$$

Lemma 3.1. If $\left(A_{1}\right)$ and $\left(A_{2}\right)$ are fulfilled, then the positive equilibrium point $\left(w_{1 *}, w_{3 *}\right)$ is locally asymptotically stable.

Proof Under the assumption $\left(A_{1}\right)$, one knows that system (5) own a unique positive equilibrium point $\left(w_{1 *}, w_{3 *}\right)$. If $\gamma=0$. Then (14) owns the form:

$$
\lambda^{2}+\left(\beta_{1}+\varsigma_{1}\right) \lambda+\beta_{2}+\varsigma_{2}=0
$$

By $\left(A_{2}\right)$, one can conclude that every root $\lambda_{i}$ of (16) satisfies $\left|\arg \left(\lambda_{i}\right)\right|>\frac{\eta \pi}{2}(i=1,2)$. It follows from Lemma 3.1 that $\left(w_{1 *}, w_{3 *}\right)$ is locally asymptotically stable, which ends the proof.

Assume that $s=i \vartheta=\vartheta\left(\cos \frac{\pi}{2}+i \sin \frac{\pi}{2}\right)$ is a root of (14). Then

$$
\left\{\begin{array}{l}
b_{1} \cos \vartheta \gamma+b_{2} \sin \vartheta \gamma=c_{1}, \\
b_{2} \cos \vartheta \gamma-b_{1} \sin \vartheta \gamma=c_{2},
\end{array}\right.
$$

where

$$
\left\{\begin{array}{l}
b_{1}=\varsigma_{1} \vartheta^{\eta} \cos \frac{\eta \pi}{2}+\varsigma_{2}, \\
b_{2}=\varsigma_{1} \vartheta^{\eta} \sin \frac{\eta^{\pi}}{2} \\
c_{1}=-\vartheta^{2 \eta} \cos \eta \pi-\beta_{1} \vartheta^{\eta} \cos \frac{\eta \pi}{2}-\beta_{2}, \\
c_{2}=-\vartheta^{2 \eta} \sin \eta \pi-\beta_{1} \vartheta^{\eta} \sin \frac{\eta \pi}{2} .
\end{array}\right.
$$

By (3.17), one gets

$$
b_{1}^{2}+b_{2}^{2}=c_{1}^{2}+c_{2}^{2}
$$

By means of (18) and (19), we have

$$
\vartheta^{4 \eta}+d_{1} \vartheta^{3 \eta}+d_{2} \vartheta^{2 \eta}+d_{3} \vartheta^{\eta}+d_{4}=0
$$


where

$$
\left\{\begin{aligned}
d_{1} & =2 \beta_{1}\left(\cos \eta \pi \cos \frac{\eta \pi}{2}+\sin \eta \pi \sin \frac{\eta \pi}{2}\right) \\
d_{2} & =\beta_{1}^{2}+2 \beta_{2} \cos \eta \pi-\varsigma_{1}^{2} \\
d_{3} & =2\left(\beta_{1} \beta_{2}-\varsigma_{1} \varsigma_{2}\right) \cos \frac{\eta \pi}{2} \\
d_{4} & =\beta_{2}^{2}-\varsigma_{2}^{2} .
\end{aligned}\right.
$$

Denote

$$
\Omega(\vartheta)=\vartheta^{4 \eta}+d_{1} \vartheta^{3 \eta}+d_{2} \vartheta^{2 \eta}+d_{3} \vartheta^{\eta}+d_{4}
$$

Assume that

$$
\left(A_{3}\right) \beta_{2}^{2}<\varsigma_{2}^{2}
$$

Under the condition $\left(A_{3}\right)$, one knows that $d_{4}<0$. In addition, $\frac{d \Omega(\vartheta)}{d \vartheta}>0, \forall \vartheta>0$, then Eq.(20) owns at least one positive real root. Thus Eq.(14) owns at least one pair of purely roots.

Assume that Eq.(15) owns four real roots $\vartheta_{k}>0(k=1,2,3,4)$. In view of (17), we obtain

$$
\gamma_{k}^{j}=\frac{1}{\vartheta_{k}}\left[\arccos \left(\frac{b_{1} c_{1}+b_{2} c_{2}}{b_{1}^{2}+b_{2}^{2}}\right)+2 j \pi\right]
$$

where $j=0,1,2, \cdots, k=1,2,3,4$. Let

$$
\gamma_{0}=\min _{k=1,2,3,4}\left\{\gamma_{k}^{0}\right\}, \vartheta_{0}=\left.\vartheta\right|_{\gamma=\gamma_{0}}
$$

Next we give the following hypothesis:

$\left(A_{4}\right) \mathcal{U}_{1} \mathcal{W}_{1}+\mathcal{U}_{2} \mathcal{W}_{2}>0$, where

$$
\left\{\begin{aligned}
\mathcal{U}_{1}= & 2 \eta \vartheta_{0}^{2 \eta-1} \cos \frac{(2 \eta-1) \pi}{2}+\eta \beta_{1} \vartheta_{0}^{\eta-1} \cos \frac{(\eta-1) \pi}{2} \\
& +\eta \varsigma_{1} \vartheta_{0}^{\eta-1} \cos \frac{(\eta-1) \pi}{2} \cos \vartheta_{0} \gamma_{0}+\eta \varsigma_{1} \vartheta_{0}^{\eta-1} \sin \frac{(\eta-1) \pi}{2} \sin \vartheta_{0} \gamma_{0}, \\
\mathcal{U}_{2}= & 2 \eta \vartheta_{0}^{2 \eta-1} \sin \frac{(2 \eta-1) \pi}{2}+\eta \beta_{1} \vartheta_{0}^{\eta-1} \sin \frac{(\eta-1) \pi}{2} \\
& -\eta \varsigma_{1} \vartheta_{0}^{\eta-1} \cos \frac{(\eta-1) \pi}{2} \sin \vartheta_{0} \gamma_{0}+\eta \varsigma_{1} \vartheta_{0}^{\eta-1} \sin \frac{(\eta-1) \pi}{2} \cos \vartheta_{0} \gamma_{0}, \\
\mathcal{W}_{1}= & \gamma\left(\varsigma_{1} \vartheta_{0}^{\eta} \cos \frac{\eta \pi}{2}+\varsigma_{2}\right) \cos \vartheta_{0} \gamma_{0}+\gamma \varsigma_{1} \vartheta_{0}^{\eta} \sin \frac{\eta \pi}{2} \sin \vartheta_{0} \gamma_{0}, \\
\mathcal{W}_{2}= & -\gamma\left(\varsigma_{1} \vartheta_{0}^{\eta} \cos \frac{\eta \pi}{2}+\varsigma_{2}\right) \sin \vartheta_{0} \gamma_{0}+\gamma \varsigma_{1} \vartheta_{0}^{\eta} \sin \frac{\eta \pi}{2} \cos \vartheta_{0} \gamma_{0} .
\end{aligned}\right.
$$

Lemma 3.2. Suppose that $s(\gamma)=\mu_{1}(\gamma)+i \mu_{2}(\gamma)$ is the root of (14) near $\gamma=\gamma_{0}$ satisfying $\mu_{1}\left(\gamma_{0}\right)=0, \mu_{2}\left(\gamma_{0}\right)=\vartheta_{0}$, then $\operatorname{Re}\left[\frac{d s}{d \gamma}\right]_{\gamma=\gamma_{0}, \vartheta=\vartheta_{0}}>0$.

Proof According to (14), we get

$$
2 \eta s^{2 \eta-1}+\eta \beta_{1} s^{\eta-1}+\eta \varsigma_{1} s^{\eta-1} e^{-s \gamma}-e^{-s \gamma}\left(\frac{d s}{d \gamma} \gamma+s\right)\left(\varsigma_{1} s^{\eta}+\varsigma_{2}\right)=0,
$$


which leads to

$$
\left(\frac{d s}{d \gamma}\right)^{-1}=\frac{2 \eta s^{2 \eta-1}+\eta \beta_{1} s^{\eta-1}+\eta \varsigma_{1} s^{\eta-1} e^{-s \gamma}}{\gamma\left(\varsigma_{1} s^{\eta}+\varsigma_{2}\right) e^{-s \gamma}}-\frac{\gamma}{s} .
$$

Hence

$$
\operatorname{Re}\left[\left(\frac{d s}{d \gamma}\right)^{-1}\right]=\operatorname{Re}\left[\frac{2 \eta s^{2 \eta-1}+\eta \beta_{1} s^{\eta-1}+\eta \varsigma_{1} s^{\eta-1} e^{-s \gamma}}{\gamma\left(\varsigma_{1} s^{\eta}+\varsigma_{2}\right) e^{-s \gamma}}\right] .
$$

Thus

$$
\begin{aligned}
\operatorname{Re}\left[\left(\frac{d s}{d \varepsilon_{2}}\right)^{-1}\right]_{\gamma=\gamma_{0}, \vartheta=\vartheta_{0}} & =\operatorname{Re}\left[\frac{2 \eta s^{2 \eta-1}+\eta \beta_{1} s^{\eta-1}+\eta \varsigma_{1} s^{\eta-1} e^{-s \gamma}}{\gamma\left(\varsigma_{1} s^{\eta}+\varsigma_{2}\right) e^{-s \gamma}}\right]_{\gamma=\gamma_{0}, \vartheta=\vartheta_{0}} \\
& =\frac{\mathcal{U}_{1} \mathcal{W}_{1}+\mathcal{U}_{2} \mathcal{W}_{2}}{\mathcal{W}_{1}^{2}+\mathcal{W}_{2}^{2}}
\end{aligned}
$$

By $\left(A_{4}\right)$, one gets

$$
\operatorname{Re}\left[\left(\frac{d s}{d \gamma}\right)^{-1}\right]_{\gamma=\gamma_{0}, \vartheta=\vartheta_{0}}>0
$$

which finishes the proof.

On the basis of discussion above, the following fruit is derived.

Theorem 3.1. Assume that $\left(A_{1}\right)-\left(A_{4}\right)$ are satisfied, then the positive equilibrium point $\left(w_{1 *}, w_{3 *}\right)$ of system (5) is locally asymptotically stable provided that $\gamma$ lies in the interval $\left[0, \gamma_{0}\right)$ and a Hopf bifurcation will arise around $\left(w_{1 *}, w_{3 *}\right)$ provided that $\gamma$ exceeds the value $\gamma_{0}$.

Remark 3.1. In [5], Wu and Zhang reported the bifurcation of integer-order Oregonator model. They do not consider the fractional-order case. In this work, based on the work of Wu and Zhang [5], we set up a novel fractional-order Oregonator model which can better describe memory and hereditary property of different chemical composition. We obtain the characteristic equation of the involved fractional-order Oregonator model by using laplace transform which are different from that for integer-order case in [5]. From this aspect, we think that the fruit of this work on the stability and the appearance of Hopf bifurcation of fractional-order Oregonator model (5) are perfectly novel and replenish the work of [5] to a certain degree.

\section{Computer simulations}

Example 4.1 Consider the following fractional-order Oregonator model:

$$
\left\{\begin{array}{l}
\epsilon \frac{d w_{1}^{\eta}(t)}{d t^{\eta}}=\frac{a g w_{3}}{a+w_{1}}-\frac{g w_{1} w_{3}}{a+w_{1}}+w_{1}\left(1-w_{1}\right), \\
\frac{d w_{3}^{\eta}(t)}{d t^{\eta}}=w_{1}-w_{3}+\theta w_{3}(t-\gamma)
\end{array}\right.
$$


where $a=0.0004, g=0.7, \theta=2.5, \epsilon=0.3$. Evidently, system (30) owns the unique positive equilibrium point $(0.8002,0.2286)$. Choose $\eta=0.72$. By means of Matlab, we get $\vartheta_{0}=0.9023$ and $\gamma_{0}=0.017$. The hypotheses $\left(A_{1}\right)-\left(A_{4}\right)$ in Theorem 3.1 hold true. In order to check the stability and Hopf bifurcation of Theorem 3.1, we choose two different time delay values. Select $\gamma=0.012<\gamma_{0}=0.017$, then the computer simulation results are shown in Figure 1 which includes four subfigures. From figure 1, one can see that the positive equilibrium point $(0.8002,0.2286)$ is locally asymptotically stable. Subfigure 1 of figure 1 shows the relation of $t$ and $w_{1}$, subfigure 2 of figure 1 shows the relation of $t$ and $w_{3}$, subfigure 3 of figure 1 shows the relation of $w_{1}$ and $w_{3}$, subfigure 4 of figure 1 shows the relation of $t, w_{1}$ and $w_{3}$. Select $\gamma=0.026>\gamma_{0}=0.017$, then the computer simulation results are shown in Figure 2 which includes four subfigures. From figure 2, one can see that the positive equilibrium point $(0.8002,0.2286)$ become unstable and a Hopf bifurcation takes place. Subfigure 1 of figure 2 shows the relation of $t$ and $w_{1}$, subfigure 2 of figure 2 shows the relation of $t$ and $w_{3}$, subfigure 3 of figure 2 shows the relation of $w_{1}$ and $w_{3}$, subfigure 4 of figure 2 shows the relation of $t, w_{1}$ and $w_{3}$. In Table 1 , we have given the relation of $\eta, \vartheta_{0}$ and $\gamma_{0}$ of (30). Also, the bifurcation plots are given to show the bifurcation value is 0.017 (see Figures 3-4).
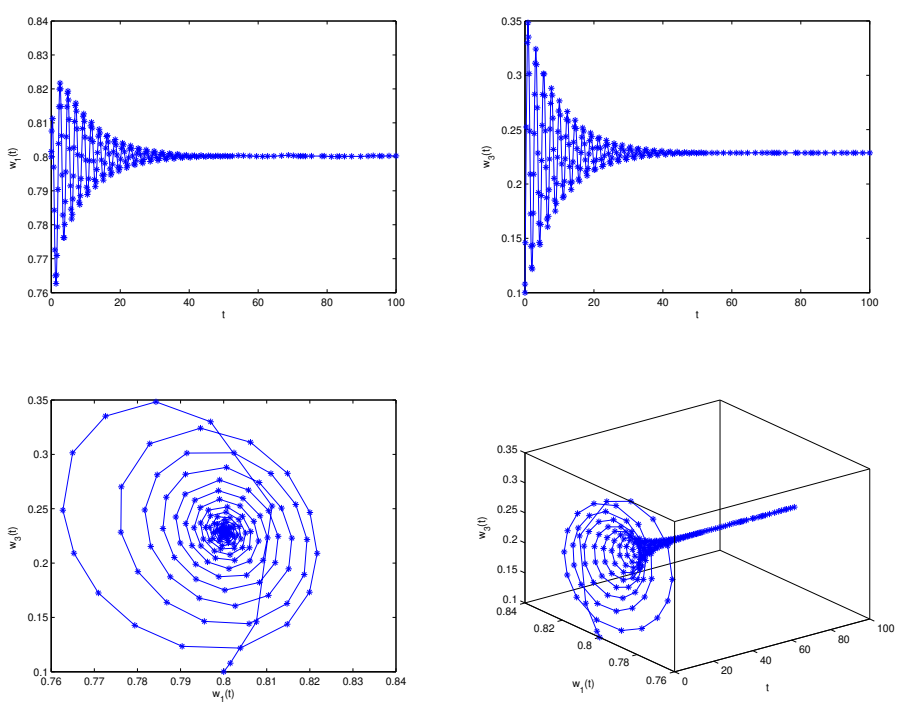

Figure 1. $\gamma=0.012<\gamma_{0}=0.017$ 

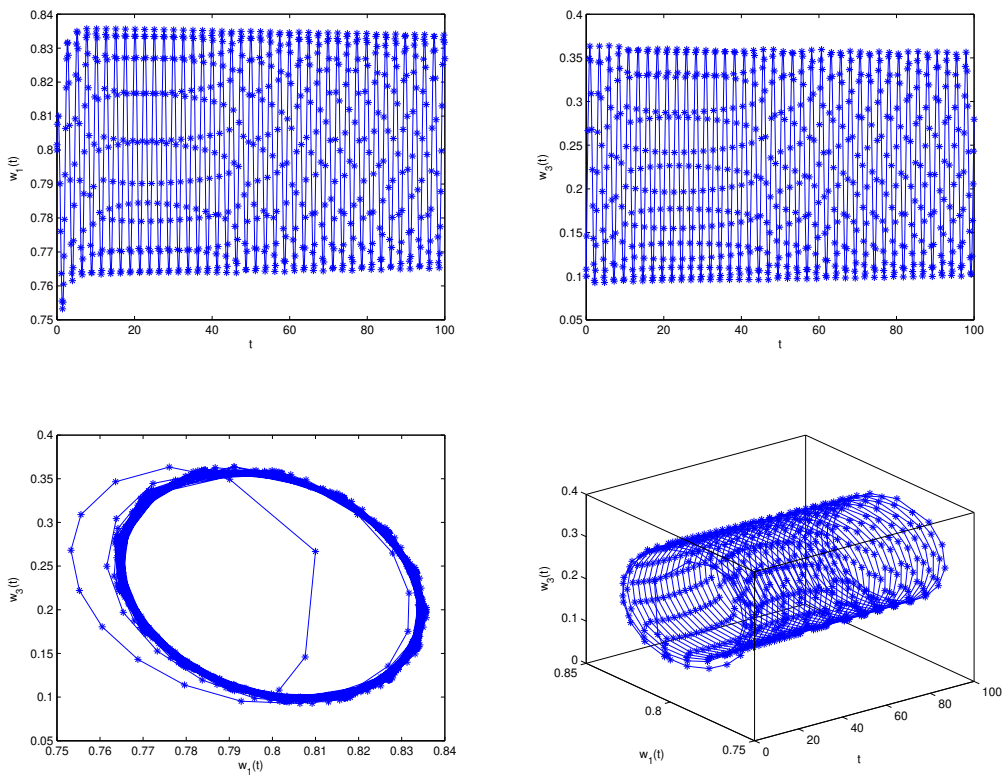

Figure 2. $\gamma=0.026>\gamma_{0}=0.017$.

Table 1. The relation of $\eta, \vartheta_{0}$ and $\gamma_{0}$ of (30).

\begin{tabular}{ccc}
\hline$\eta$ & $\vartheta_{0}$ & $\gamma_{0}$ \\
\hline 0.13 & 1.5322 & 0.005 \\
0.25 & 1.4055 & 0.008 \\
0.31 & 1.3744 & 0.01 \\
0.42 & 1.2056 & 0.012 \\
0.50 & 1.1056 & 0.015 \\
0.62 & 1.0231 & 0.018 \\
0.72 & 0.9023 & 0.017 \\
0.81 & 0.7245 & 0.042 \\
0.92 & 0.6723 & 0.051 \\
\hline
\end{tabular}




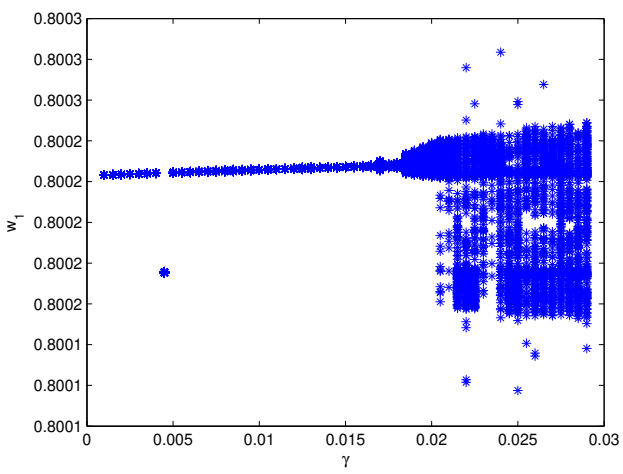

Figure 3. Bifurcation plot of Oregonator system (30): $\gamma-w_{1}$.

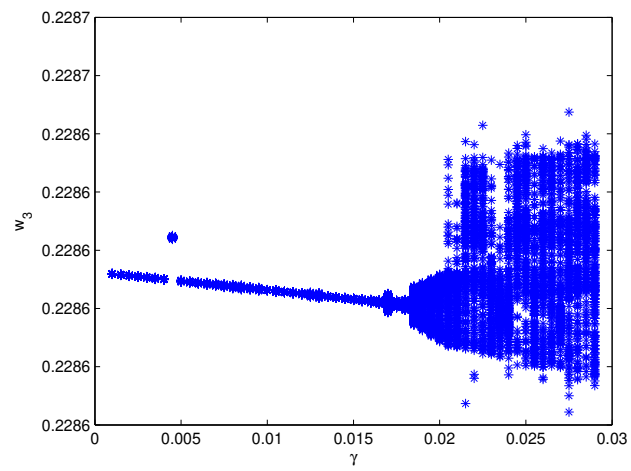

Figure 4. Bifurcation plot of Oregonator system (30): $\gamma-w_{3}$.

Example 4.2 Consider the following fractional-order Oregonator model:

$$
\left\{\begin{array}{l}
\epsilon \frac{d w_{1}^{\eta}(t)}{d t^{\eta}}=\frac{a g w_{3}}{a+w_{1}}-\frac{g w_{1} w_{3}}{a+w_{1}}+w_{1}\left(1-w_{1}\right), \\
\frac{d w_{3}^{\eta}(t)}{d t^{\eta}}=w_{1}-w_{3}+\theta w_{3}(t-\gamma),
\end{array}\right.
$$

where $a=0.00035, g=0.9, \theta=2.6, \epsilon=0.35$. Evidently, system (31) owns the unique positive equilibrium point $(0.7502,0.2084)$. Choose $\eta=0.76$. By means of Matlab, we get $\vartheta_{0}=.6187$ and $\gamma_{0}=0.010$. The hypotheses $\left(A_{1}\right)-\left(A_{4}\right)$ in Theorem 3.1 hold true. In order to check the stability and Hopf bifurcation of Theorem 3.1, we choose two different time delay values. Select $\gamma=0.008<\gamma_{0}=0.010$, then the computer simulation results are shown in Figure 5 which includes four subfigures. From figure 5, one can see that 
the positive equilibrium point $(0.7502,0.2084)$ is locally asymptotically stable. Subfigure 1 of figure 5 shows the relation of $t$ and $w_{1}$, subfigure 2 of figure 5 shows the relation of $t$ and $w_{3}$, subfigure 3 of figure 5 shows the relation of $w_{1}$ and $w_{3}$, subfigure 4 of figure 5 shows the relation of $t, w_{1}$ and $w_{3}$. Select $\gamma=0.015>\gamma_{0}=0.010$, then the computer simulation results are shown in Figure 6 which includes four subfigures. From figure 6 , one can see that the positive equilibrium point $(0.7502,0.2084)$ become unstable and a Hopf bifurcation takes place. Subfigure 1 of figure 6 shows the relation of $t$ and $w_{1}$, subfigure 2 of figure 6 shows the relation of $t$ and $w_{3}$, subfigure 3 of figure 6 shows the relation of $w_{1}$ and $w_{3}$, subfigure 4 of figure 6 shows the relation of $t, w_{1}$ and $w_{3}$. In Table 2 , we have given the relation of $\eta, \vartheta_{0}$ and $\gamma_{0}$ of (31). Also, the bifurcation plots are given to show the bifurcation value is 0.010 (see Figures 7-8).
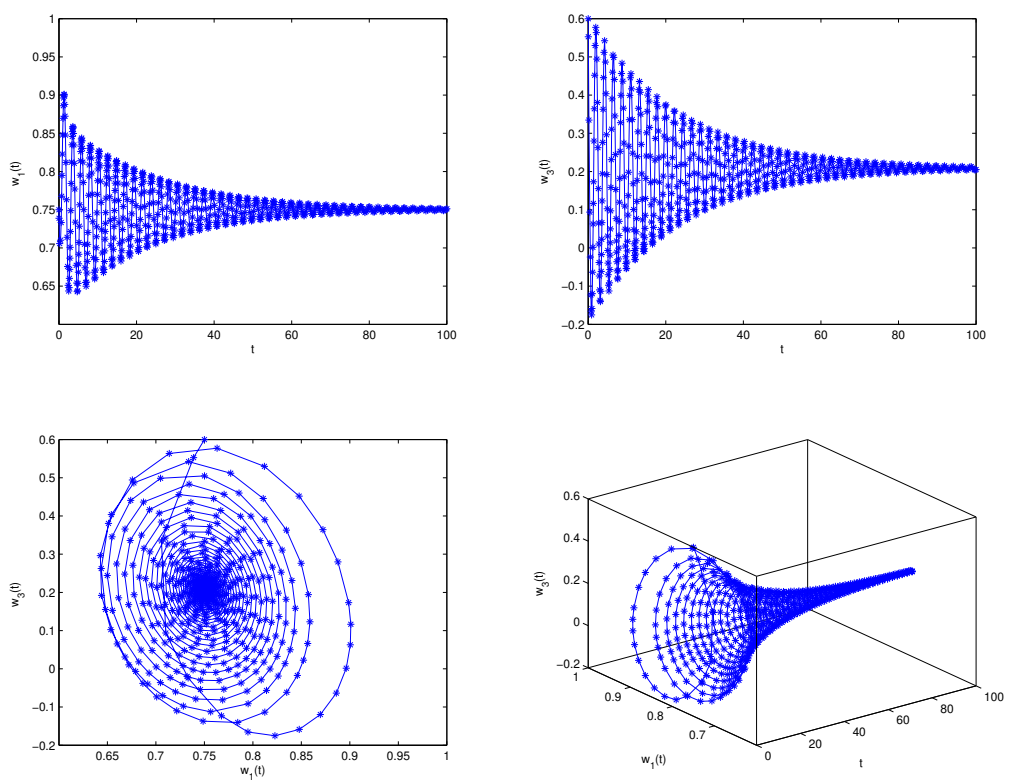

Figure 5. $\gamma=0.008<\gamma_{0}=0.010$ 

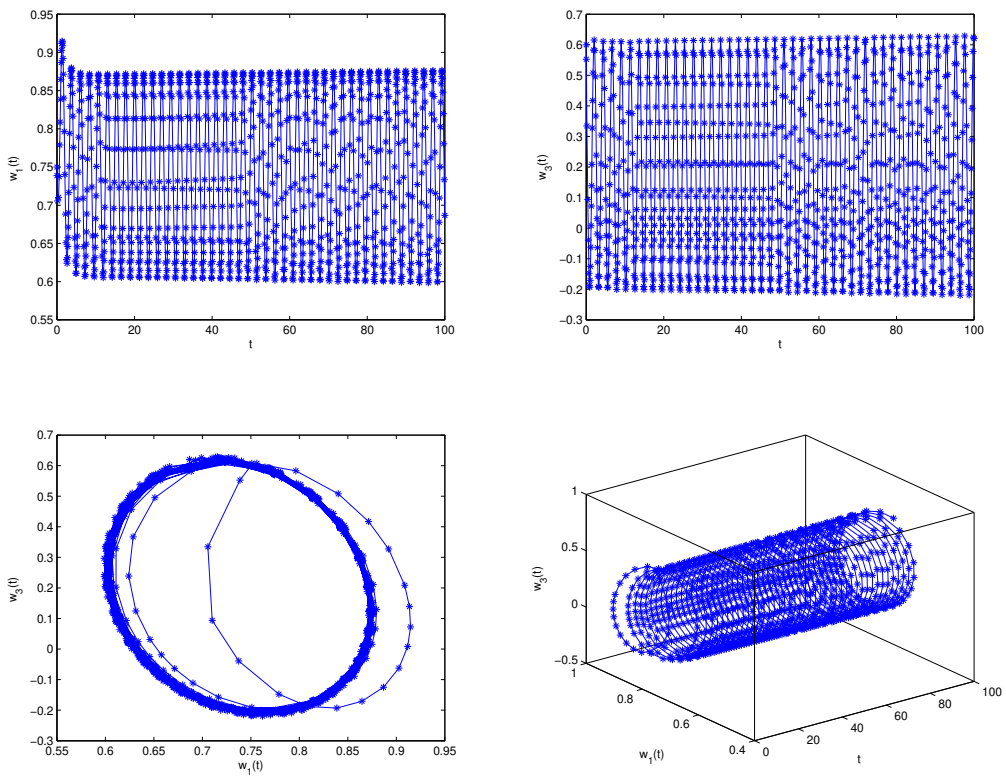

Figure 6. $\gamma=0.015>\gamma_{0}=0.010$.

Table 2. The relation of $\eta, \vartheta_{0}$ and $\gamma_{0}$ of (31).

\begin{tabular}{ccc}
\hline$\eta$ & $\vartheta_{0}$ & $\gamma_{0}$ \\
\hline 0.12 & 1.3409 & 0.00012 \\
0.24 & 1.2352 & 0.00018 \\
0.32 & 1.1203 & 0.00021 \\
0.43 & 0.9122 & 0.00025 \\
0.51 & 0.8132 & 0.00500 \\
0.63 & 0.7023 & 0.00800 \\
0.75 & 0.6187 & 0.01000 \\
0.82 & 0.6022 & 0.01200 \\
0.93 & 0.5835 & 0.01500 \\
\hline
\end{tabular}




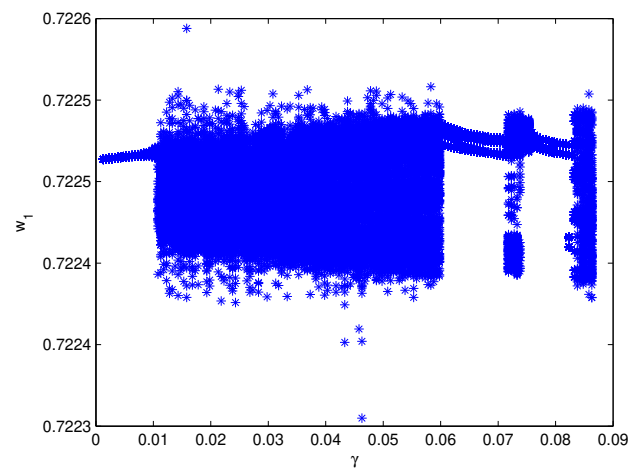

Figure 7. Bifurcation plot of Oregonator system (31): $\gamma-w_{1}$.

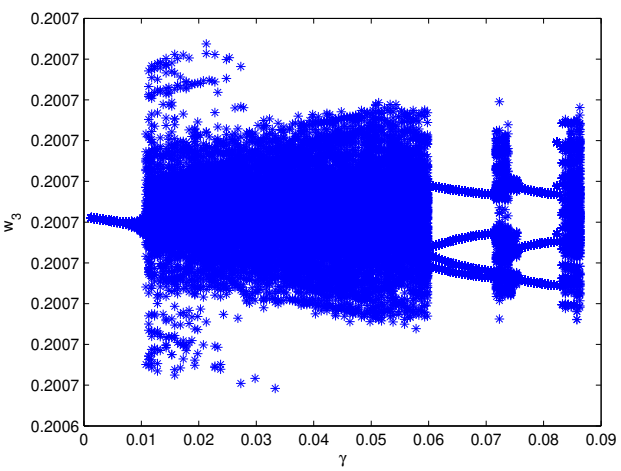

Figure 8. Bifurcation plot of Oregonator system (31): $\gamma-w_{3}$.

\section{Conclusions}

Constructing mathematical models to portray the relation of different chemical compositions is an important topic in chemistry. However, many works on this aspect are concerned with the construct of integer-order differential equation models, very few works involve the fractional-order chemical models. Based on the earlier works, we set up a novel fractional-order Oregonator model including time delay. Applying Laplace transform, we obtain the characteristic equation of the involved fractional-order delayed Oregonator model. By regarding the time delay as bifurcation parameter, we establish a new delayindependent stability and bifurcation condition to guarantee the stability and the emer- 
gence of Hopf bifurcation for the considered Oregonator model. The investigation shows that if the time delay lies in a suitable suitable interval, the Oregonator system will keep a locally stable level and when the time delay exceeds some values, the equilibrium point of the Oregonator system will become unstable and a Hopf bifurcation will take place. It also reveals that time delay plays a vital role in controlling the bifurcation behavior of the fractional-order delayed Oregonator system. The derived fruits are helpful to control the concentrations of $\mathrm{HBrO}_{2}, \mathrm{Br}^{-}$and $\mathrm{Ce}(\mathrm{IV})$. In addition, the research fruits of this paper supplement the work of $\mathrm{Wu}$ and Zhang [5].

Acknowledgments: This work is supported by National Natural Science Foundation of China (No.61673008 and No.62062018) and Project of High-level Innovative Talents of Guizhou Province ([2016]5651).

\section{References}

[1] B. P. Belousov, R. J. Field, M. Burger, Oscillations and Traveling Waves in Chemical Systems, Wiley, New York, 1985, 605-613.

[2] S. P. Hastings, J. D. Murray, The existence of oscillatory solutions in the FieldNoyes model for the Belousov-Zhabotinskii reaction, SIAM J. Appl. Math. 28 (1975) 678-688.

[3] C. Mirase, S. Sakanoue, M. Endo, Oregonator model with a flow term for the Belousov Zhabotinskii reaction having constant feed. II: Oregonator model with a constant flow term, Prog. Theor. Phy. 77 (1987) 1319-1333.

[4] P. Gray, S. K. Scott, Chemical Oscillations and Instabilities, Oxford Univ. Press, Oxford, 1994.

[5] X. Wu, C. R. Zhang, Dynamic properties of the Oregonator model with delay, J. Appl. Anal. Comput. 2 (2012) 91-102.

[6] X. J. Yang, C. D. Li, Q. K. Song, J. Y. Chen, J. J. Huang, Global Mittag-Leffler stability and synchronization analysis of fractional-order quaternion-valued neural networks with linear threshold neurons, Neural Netw. 105 (2018) 88-103.

[7] J. J. Chen, B. S. Chen, Z. G. Zeng, $O\left(t^{-\alpha}\right)$-synchronization and Mittag-Leffler synchronization for the fractional-order memristive neural networks with delays and discontinuous neuron activations, Neural Netw. 100 (2018) 10-24. 
[8] C. J. Xu, Z. X. Liu, L. Y. Yao, C. Aouiti, Further exploration on bifurcation of fractional-order six-neuron bi-directional associative memory neural networks with multi-delays, Appl. Math. Comput. 410 (2021) \#126458.

[9] Q. H. Feng, F. W. Meng, Traveling wave solutions for fractional partial differential equations arising in mathematical physics by an improved fractional Jacobi elliptic equation method, Math. Meth. Appl. Sci. 40 (2017) 3676-3686.

[10] F. H. Zhang, T. W. Huang, Q. J. Wu, Z. G. Zeng, Multistability of delayed fractionalorder competitive neural networks, Neural Netw. 140 (2021) 325-335.

[11] N. Padmaja, P. Balasubramaniam, New delay and order-dependent passivity criteria for impulsive fractional-order neural networks with switching parameters and proportional delays, Neurocomputing 454 (2021) 113-123.

[12] F. B. Yousef, A. Yousef, C. Maji, Effects of fear in a fractional-order predator-prey system with predator density-dependent prey mortality, Chaos Soliton Fract. 145 (2021) \#110711.

[13] B. Ghanbari, S. Djilali, Mathematical analysis of a fractional-order predator-prey model with prey social behavior and infection developed in predator population, Chaos Soliton Fract. 138 (2020) \#109960.

[14] C. J. Xu, Z. X. Liu, M. X. Liao, P. L. Li, Q. M. Xiao, S. Yuan, Fractional-order bidirectional associate memory (BAM) neural networks with multiple delays: The case of Hopf bifurcation, Math. Comput. Simul. 182 (2021) 471-494.

[15] J. Yuan, L. Z. Zhao, C. D. Huang, M. Xiao, Stability and bifurcation analysis of a fractional predator-prey model involving two nonidentical delays, Math. Comput. Simul. 181 (2021) 562-580.

[16] S. Eshaghi, R. K. Ghaziani, A. Ansari, Hopf bifurcation, chaos control and synchronization of a chaotic fractional-order system with chaos entanglement function, Math. Comput. Simul. 172 (2020) 321-340.

[17] C. D. Huang, J. D. Cao, M. Xiao, Hybrid control on bifurcation for a delayed fractional gene regulatory network, Chaos Soliton Fract. 87 (2016) 19-29.

[18] A. S. Deshpande, V. D. Gejji, Y. V. Sukale, On Hopf bifurcation in fractional dynamical systems, Chaos Soliton Fract. 98 (2017) 189-198.

[19] C. J. Xu, C. Aouiti, Comparative analysis on Hopf bifurcation of integer order and fractional order two-neuron neural networks with delay, Int. J. Circ. Theor. Appl. 48 (2020) 1459-1475. 
[20] C.D. Huang, Multiple scales scheme for bifurcation in a delayed extended van der Pol oscillator, Phys. A 490 (2018) 643-652.

[21] M. Xiao, W. X. Zheng, J. X. Lin, G. P. Jiang, L. D. Zhao, Fractional-order PD control at Hopf bifurcation in delayed fractional-order small-world networks, J. Franklin Inst. 354 (2017) 7643-7667.

[22] M. Xiao, G. P. Jiang, W. X. Zheng, S. L. Yan, Y. H. Wan, C. X. Fan, Bifurcation control od a fractional-order van der pol oscillator based on the state feedback, Asian J. Control 17 (2015) 1755-1766.

[23] C. J. Xu, C. Aouiti, Z. X. Liu, A further study on bifurcation for fractional order BAM neural networks with multiple delays, Neurocomputing 417 (2020) 501-515.

[24] C. J. Xu, M. X. Liao, P. L. Li, Y. Guo, Z. X. Liu, Bifurcation properties for fractional order delayed BAM neural networks, Cogn. Comput. 13 (2021) 322-356.

[25] C. J. Xu, M. X. Liao, P. L. Li, S. Yuan, Impact of leakage delay on bifurcation in fractional-order complex-valued neural networks, Chaos Soliton Fract. 142 (2021) \#110535.

[26] I. Podlubny, Fractional Differential Equations, Academic Press, New York, 1999.

[27] C. D. Huang, J. D. Cao, M. Xiao, A. Alsaedi, T. Hayat, Bifurcations in a delayed fractional complex-valued neural network, Appl. Math. Comput. 292 (2017) 210-227.

[28] D. Matignon, Stability results for fractional differential equations with applications to control processing, in: Computational Engineering in Systems Applications, IEEESMC Proceedings, Lille, 1996, pp. 963-968.

[29] W. H. Deng, C. P. Li, J. H. Lü, Stability analysis of linear fractional differential system with multiple time delays, Nonlin. Dyn. 48 (2007) 409-416. 